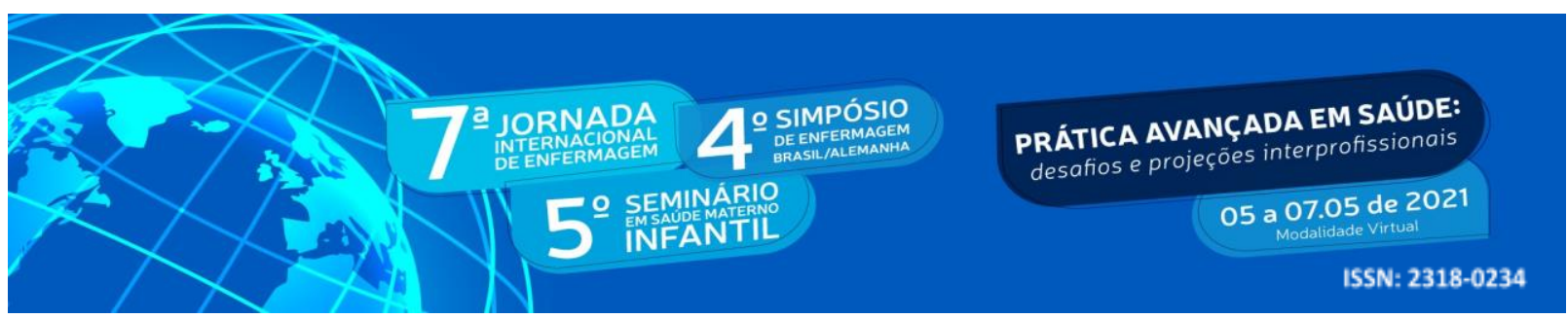

DOI: http://doi.org/10.48195/jie2021-115

\title{
O PAPEL DA ENFERMAGEM FRENTE A DIABETES MELLITUS GESTACIONAL ${ }^{1}$
}

\author{
Munah Najeh Saleh Ahmad Maruf²; Claudia Maria Gabert Diaz ${ }^{3}$; Gicelle Moraes \\ Martelli $^{4}$; Carolina Londero Araujo ${ }^{5}$; Kelvin Leandro Marques Monçalves ${ }^{6}$; Stéphani

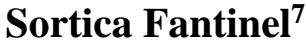

\begin{abstract}
RESUMO
O principal objetivo deste estudo é ampliar o conhecimento sobre Diabetes Gestacional, assim como o papel do cuidado de enfermagem frente a mesma, enfatizando a importância do pré-natal, promoção e prevenção da saúde. Trata-se de um estudo bibliográfico consultado em materiais científicos, disponíveis na Biblioteca Virtual da Saúde, nas bases Scielo e Lilacs. Durante o período gestacional o corpo da mulher passa por diversas alterações hormonais, que podem ocasionar o aumento de glicemia, podendo causar complicações ao sistema renal e sistêmico. As complicações fetais e neonatais são decorrentes dos níveis glicêmicos descompensados, que podem desencadear aborto espontâneo, malformações congênitas e macrossomia. Conclui-se que a Atenção Primária possui grande responsabilidade, visto que é a porta de entrada do sistema de saúde. Portanto, é dever do profissional, da gestante e acompanhante realizar o pré-natal, para então identificar fatores de risco, garantindo uma gestação segura e com todo apoio e acolhimento necessário.
\end{abstract}

Palavras-chave: Cuidado de Enfermagem; Diabetes Gestacional; Diagnóstico Pré-Natal.

\footnotetext{
${ }^{1}$ Trabalho de pesquisa realizado na disciplina de Saúde da Mulher do $6^{\circ}$ semestre do curso de enfermagem, Universidade Franciscana 2020.

${ }^{2}$ Acadêmica do curso de enfermagem-UFN. E-mail: munahsaleh1999@gmail.com

${ }^{3}$ Orientadora. Doutora em enfermagem. Docente do curso de enfermagem-UFN. E-mail: cmgdiaz@ufn.edu.br

${ }^{4}$ Acadêmica do curso de enfermagem-UFN. E-mail: gicelle1219@gmail.com

${ }^{5}$ Acadêmica do curso de enfermagem-UFN. E-maik: carolina.alondero@gmail.com

${ }^{6}$ Acadêmico do curso de enfermagem-UFN. E-mail: marqueskelvin5@gmail.com

${ }^{7}$ Acadêmica do curso de enfermagem-UFN. E-mail: stephanifantinel@gmail.com
} 


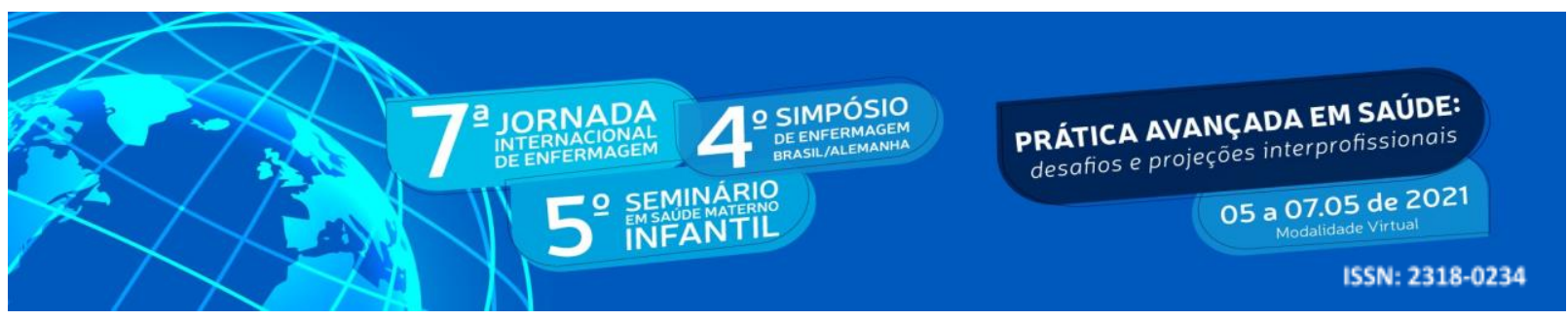

\begin{abstract}
The main objective of this study is to expand knowledge about Gestational Diabetes, as well as the role of nursing care in face off it, emphasizing the importance of prenatal care, health promotion and prevention. It is a bibliographic study consulted in scientific materials, available in the Virtual Health Library, in the Scielo and Lilacs databases. During the gestational period, the woman's body undergoes several hormonal changes, which can cause as increase in blood glucose, which can cause complications are due to decompensated glycemic level, as it can trigger a spontaneous abortion, congenital malformations and macrosomia. It is concluded that Primary care has great possibility, since it is the gateway to health system. Therefore, it is the duty of the professional, the pregnant woman and the companion to perform prenatal care, in order to then identify risk factor, ensuring a safe pregnancy and with all necessary support and welcome.
\end{abstract}

Key Words: Nursing care; Gestational Diabetes; Prenatal diagnosis.

\title{
1. INTRODUÇÃO
}

O Diabetes Mellitus Gestacional (DMG) ocorre frequentemente nas gestantes, sendo considerado um problema de saúde pública, que pode prejudicar a saúde da mãe e do feto (BRASIL, 2019). É uma doença que apresenta disfunção metabólica, definido como “intolerância aos carboidratos, de graus variados de intensidade, diagnosticada pela primeira vez durante a gestação, podendo ou não persistir após o parto”(BRASIL, 2012).

Esta doença é caracterizada pelo aumento da glicemia durante a gravidez, podendo causar complicações ao sistema renal e sistêmico, com a possibilidade de ocorrer até mesmo um parto antes do período desejado, com recém nascido prematuro e risco de icterícia. (BRASIL, 2019).

O organismo passa a produzir maior quantidade de insulina, que é responsável por levar a glicose proveniente dos alimentos ingeridos até as células. No terceiro trimestre da gestação, esta situação passa a ocorrer com maior intensidade, que é quando a mulher precisa ingerir maior quantidade de carboidratos para o desenvolvimento do feto (BRASIL, 2019).

Esta é uma doença de elevada incidência e deve ser identificada pelos profissionais durante o pré-natal, diminuindo os riscos materno fetais. Contudo, possui métodos para controlar e tratar a mesma. Com o fim da fase gestacional as alterações metabólicas regridem e o organismo da mulher, na maioria das vezes, volta a taxas glicêmicas normais (BRASIL, 2019). 


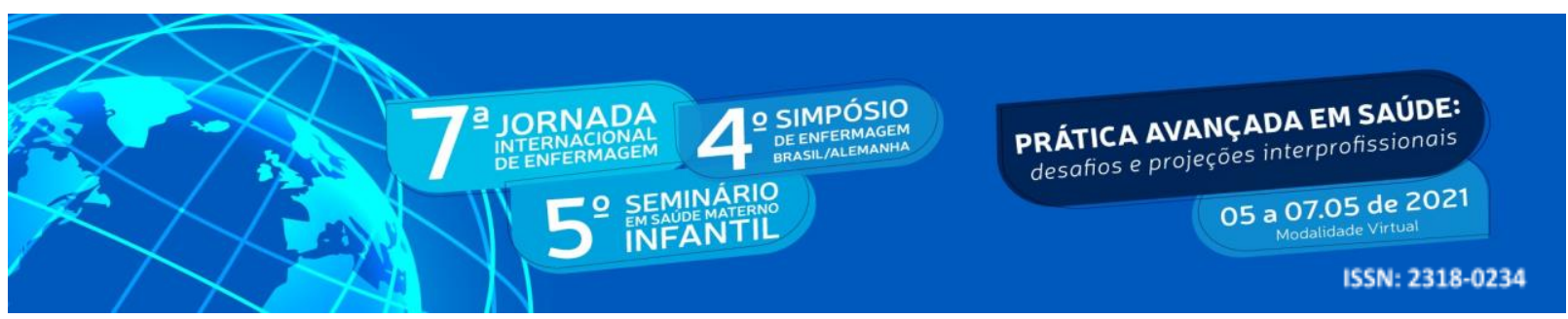

\section{OBJETIVO}

O objetivo deste estudo é ampliar o conhecimento sobre Diabetes Gestacional, assim como o papel do cuidado de enfermagem frente a mesma, enfatizando a importância do pré-natal, promoção e prevenção da saúde.

Este tema é de extrema relevância pois ocorre com incidência elevada, colocando em risco o bem-estar da gestante e seu bebê. Perpassa pelo cuidado da enfermagem e a necessidade de qualidade assistencial no pré-natal para a promoção da saúde e prevenção de agravos materno fetais, bem como a adesão terapêutica da gestante às orientações.

\section{METODOLOGIA}

Este respectivo estudo bibliográfico, de natureza teórico-reflexivo, cuja fundamentação baseia-se na formulação discursiva acerca da temática. Foi desenvolvido durante a disciplina de Atenção Integral à Saúde da Mulher, do Curso de Enfermagem, no segundo semestre de 2020. Consultado em materiais científicos encontrados na Biblioteca Virtual da Saúde, nas bases de dados Scielo e Lilacs, relativos a Diabetes Gestacional.

\section{RESULTADOS E DISCUSSÃO}

No período gestacional a mulher passa por diversas alterações, algumas visíveis e outras não visíveis aos olhos humanos, além de ser uma situação completamente nova e muitas vezes desconhecida, o medo e a insegurança são constantemente presentes em alguns períodos, assim como situações inesperadas, o que faz com que as gestantes tenham seus níveis de estresse muito elevados (JUNIOR et al, 2016).

A fisiopatologia ocorre pelas variações hormonais que acontecem no organismo durante a gestação. O corpo passa a produzir maior quantidade de insulina, contudo, os hormônios liberados pela placenta acabam prejudicando a fisiologia da insulina que é transportar a glicose até as células, fazendo com que o pâncreas trabalhe dobrado para conseguir manter os níveis da mesma como deveriam, porém, nem sempre conseguem produzir de forma 


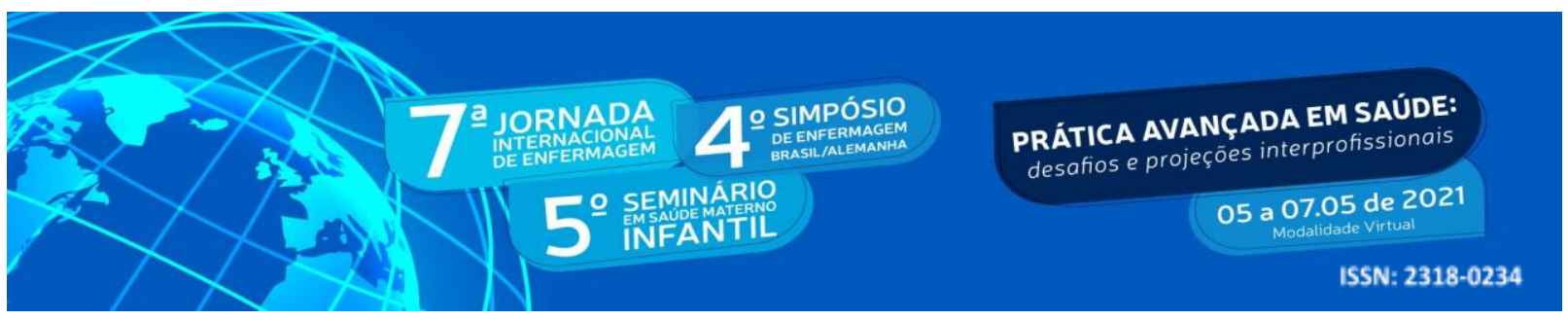

satisfatória, liberando então, glicose na corrente sanguínea, ocasionando a doença (BRASIL 2019).

A Diabetes gestacional pode ser uma destas situações inesperadas, se manifesta pela intolerância aos carboidratos em graus variados de intensidade e é diagnosticada pela primeira vez durante a gestação, podendo ou não continuar após o período gestacional. Muitas vezes a doença pode ser silenciosa, então quando descoberta acaba gerando medo a gestante, podendo então, elevar ainda mais os níveis glicêmicos, com isso, vale ressaltar a importância de realizar o tratamento solicitado (JUNIOR et al, 2016).

O rastreamento de diabetes mellitus gestacional inicia-se no pré-natal, inicialmente pela anamnese e identificação de gestantes que são diagnosticadas como grupos de risco, se encaixam as que possuem idade igual ou superior a 35 anos; com índice de massa corporal (IMC) $>25 \mathrm{~kg} / \mathrm{m} 2$ (sobrepeso e obesidade); antecedente pessoal de diabetes gestacional; antecedente familiar de Diabetes Mellitus (parentes de primeiro grau); macrossomia ou pilihidrâmnio em gestações anteriores; óbito fetal sem causa aparente em gestação anterior, malformação fetal em gestação anterior; uso de drogas hiperglicemiantes (corticoides, diuréticos e tiazídicos); síndrome dos ovários policísticos; hipertensão arterial crônica (JUNIOR et al, 2016).

$\mathrm{Na}$ atenção básica a equipe deve atentar para casos de gestantes já diagnosticadas com a doença, e no caso de gravidez planejada de uma diabética deve-se encaminhar para centros de atenção secundária ou terciária. Deve-se analisar a compensação metabólica, avaliação da presença de complicações crônicas da doença e orientação especializada para prevenção de malformações fetais, incluindo o uso rotineiro de ácido fólico. Quanto mais descompensado e alto for o nível glicêmico, mais altas serão as chances de malformações (JUNIOR et al, 2016).

É importante salientar que todas as gestantes, independentemente de serem fatores de risco ou não, devem realizar uma dosagem de glicemia no início da gravidez, no primeiro semestre ou antes da vigésima semana. Se o valor estiver abaixo de $92 \mathrm{mg} / \mathrm{dL}$, descarta-se alguma alteração neste período, porém, entre a $24^{\circ}$ e $28^{\circ}$ semana, a mulher deve realizar o exame da curva glicêmica, conhecido por TOTG. A partir deste período gestacional que a 


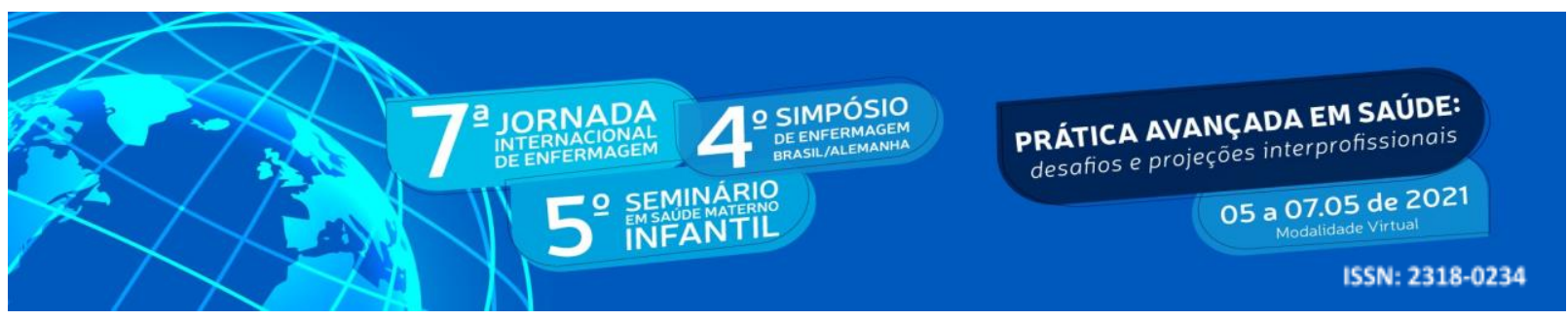

produção de hormônios contra insulínicos pela placenta é suficiente para gerar aumento da resistência periférica à insulina, ocorrendo um teste da função pancreática da gestante. Caso a glicemia de jejum esteja entre $92 \mathrm{mg} / \mathrm{dL}$ e $126 \mathrm{mg} / \mathrm{dL}$, a gestante é classificada como portadora de Diabetes Mellitus Gestacional. Se o resultado for maior ou igual a 126mg/dL, ela é classificada como Diabetes Mellitus prévio (BRASIL, 2019).

Entre todos os malefícios que podem ser ocasionados por esta doença, destacam-se agravos que podem acometer os fetos, como abortos espontâneos, nados-mortos, malformações congênitas, macrossomia, excesso de peso, hipertensão e dislipidemia. Porém, atualmente através de estudos, sabe-se que o controle glicêmico materno desempenha um fator influente nestes casos de morbilidade e que pode ser prevenido com o pré-natal (COSTA et al, 2015).

As complicações fetais e neonatais por Diabetes Mellitus são por conta dos níveis glicêmicos descompensados, pois uma hiperglicemia na gestante pode vir a desencadear uma hiperglicemia no feto, uma vez que, a glicose tem a capacidade de atravessar a placenta, porém, a insulina materna não é capaz de atravessar a mesma. Vale ressaltar que antes da $20^{\circ}$ semana as células beta do pâncreas não se encontram funcionantes, portanto não ocorre a secreção de insulina, podendo o feto estar sujeito a hiperglicemia. Após a $20^{\mathrm{a}}$ semana o feto tem o pâncreas produzindo insulina e apto para regular a homeostasia glicêmica. A hiperglicemia fetal leva à hipertrofia dos ilhéus pancreáticos fetais e consequentemente à hiperinsulinemia (COSTA et al, 2015).

Considerando as complicações que podem ocorrer, o cuidado a estas gestantes deve iniciar desde a primeira consulta de pré-natal, onde o profissional deve fazer uma boa e criteriosa avaliação, detectando possíveis doenças e orientando sobre educação em saúde e meios de preveni-las. Gestantes com o diagnóstico de Diabetes Mellitus gestacional, na maioria das vezes são encaminhadas para um pré-natal especializado no segundo ou terceiro trimestre (DIAS et al, 2019).

$\mathrm{O}$ profissional de enfermagem incumbido dos cuidados a serem prestados à esta gestante deve inserir a educação em saúde no seu cotidiano de trabalho, de forma a realçar o autocuidado da mulher. Ademais, vale ressaltar a importância da dieta nutricional, salientando os benefícios que traz ao controle da doença e do organismo (DIAS et al, 2019). 


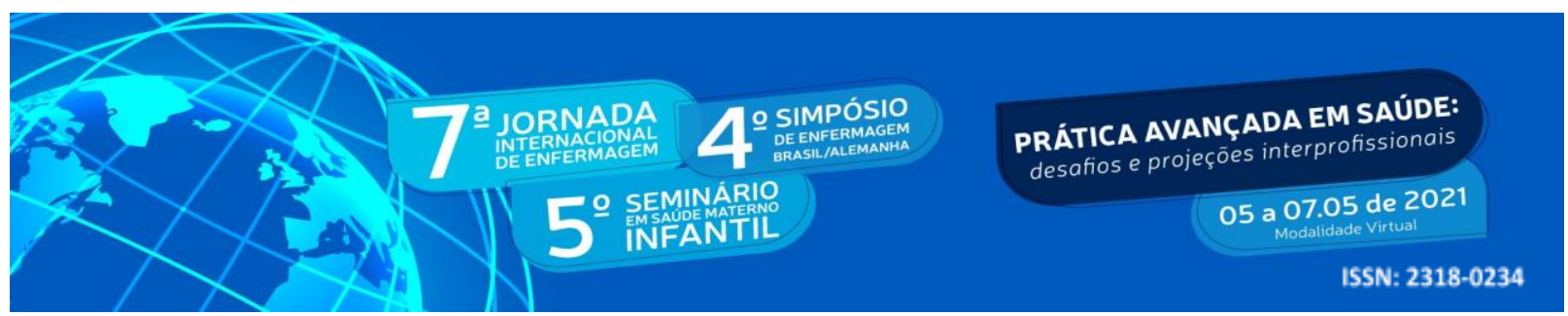

No tratamento, o enfermeiro tem um papel constante no acompanhamento desta gestante, desde o momento de identificar uma possível doença, até o encaminhamento e planejamento do plano de cuidado desta mulher da melhor forma possível, para que ela consiga colocar em prática o que lhe foi prescrito (PEREIRA et al, 2016).

Deve constar no plano de cuidados orientações educacionais como dieta nutricional, para manter o controle do peso e ter uma alimentação saudável. Ajudar em questões como encorajamento e empoderamento, para que a gestante possa e consiga realizar a monitoração diária, e a prática de exercício físico. Deve-se explicar sobre a adesão a medicamentos, se tal for prescrita, e realizar educação em saúde, para que possa vivenciar este momento com toda ajuda possível, e então, ter uma gestação tranquila e saudável (PEREIRA et al, 2016).

\section{CONCLUSÃO}

Com a realização deste trabalho, conclui-se que é de grande importância a assistência pré-natal, sendo de 6 a 8 consultas, intercaladas entre enfermeiros e médicos, dos bons hábitos alimentares e de ter uma vida saudável, visto que esta doença oferece grandes riscos à mulher e ao feto.

A Atenção Primária em Saúde (APPS) possui grande parcela de responsabilidade nesta questão, visto que é a principal porta de entrada para os usuários, e onde ocorrem as consultas de pré-natal. É dever do profissional realizar todos os exames necessários, acompanhamento e orientações, proporcionando rodas de conversa e grupos de apoio entre as gestantes e profissionais do serviço de saúde. Assim também como acolhimento e apoio durante todo período gestacional e puerperal.

Deve ser esclarecido à mulher e acompanhante a importância de obter bons hábitos alimentares, da realização de atividades físicas, caso não haja contraindicações. Junto a estas orientações, deve ser solicitado que a mesma evite ingerir alimentos ricos em lipídios, carboidratos e glicose, que foque e priorize ingerir frutas, legumes, vegetais e temperos naturais, atentando sempre ao que pode ser possível na questão econômica da família. 


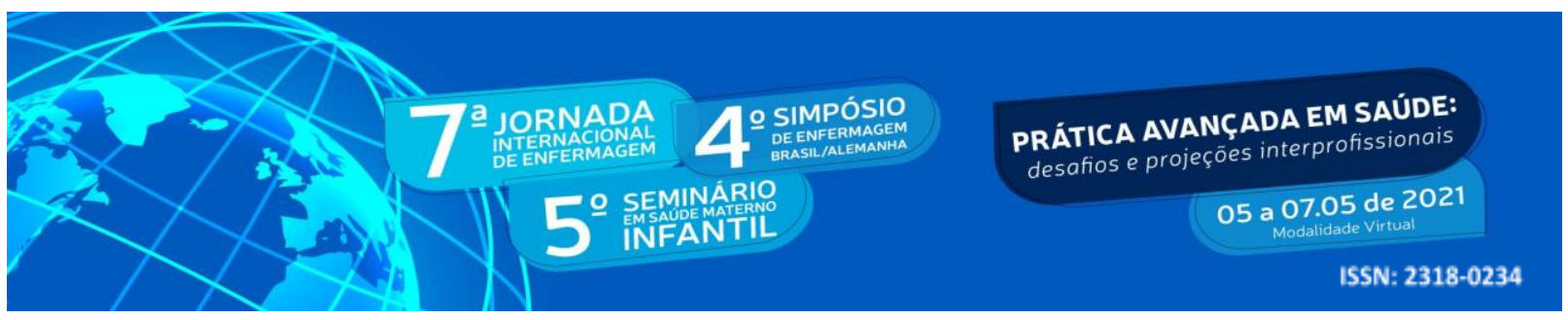

\section{REFERÊNCIAS}

BRASIL. Ministério da Saúde. Secretaria de Atenção à Saúde. Departamento de Ações Programáticas Estratégicas. Gestação de alto risco: manual técnico / Ministério da Saúde,

Secretaria de Atenção à Saúde, Departamento de Ações Programáticas Estratégicas. - 5. ed. Brasília : Editora do Ministério da Saúde, 2012. 302 p. - (Série A. Normas e Manuais Técnicos).

BRASIL. Ministério da Saúde. Secretaria de Atenção à Saúde. DIABETES GESTACIONAL. Brasília, DF, 2019. Disponível em: https://pebmed.com.br/precisamos-de-criterios-diagnosticos-mais-rigorosos-para-o-diabetes-g estacional/.

COSTA R. C.et al. Diabetes Gestacional assistida: perfil e conhecimento das gestantes. Revista Saúde (Santa Maria), v. 41, n. 1, p. 131-140, 2015. Disponível em: https://periodicos.ufsm.br/revistasaude/article/view/13504.

DIAS, G. T. et al. Cuidados de enfermagem prestados a gestantes com diabetes mellitus gestacional. 2019. Disponível em:

https://doity.com.br/media/doity/submissoes/5da4fbab-e5f4-4d42-bbea-679043cda1d7-templa te-para-envio-dmgpdf.pdf

MOREIRA, M. I. S. Diabetes Mellitus e o risco de malformações fetais. Portugal, 2015. Disponível em: file:///C:/Users/user/Downloads/Tese.pdf.

PEREIRA, F. C. et al. Cuidados de enfermagem na consulta de pré natal a gestante diagnosticada com diabetes gestacional. Revista Humano Ser - UNIFACEX, Natal-RN, v.1, n.1, p. 13-23, 2016. file://C:/Users/user/Downloads/798-2112-3-PB\%20(1).pdf.

SILVA JUNIOR, J. R. da et al . Diabetes mellitus gestacional: importância da produção de conhecimento. Rev. Bras. Saude Mater. Infant., Recife, v. 16, n. 2, p. 85-87, 2016. Disponível em: http://www.scielo.br/scielo.php?script=sci_arttext\&pid=S1519-38292016000200085\&lng=en \&nrm=iso. 